\title{
Risk factors for ectopic pregnancy: a multi- center case-control study
}

\author{
Cheng $\mathrm{Li}^{1,2+}$, Wei-Hong Zhao ${ }^{1 \dagger}$, Qian Zhu ${ }^{1 \dagger}$, Shu-Jun Cao ${ }^{3}$, Hua Ping ${ }^{4}$, Xiaowei Xi ${ }^{5}$, Guo-Juan Qin ${ }^{6}$, Ming-Xing Yan ${ }^{1}$, \\ Duo Zhang ${ }^{1}$, Jun Qiu ${ }^{1 *}$ and Jian Zhang ${ }^{1,2^{*}}$
}

\begin{abstract}
Background: Ectopic pregnancy (EP) is the leading cause of maternal death during the first trimester of pregnancy. A better understanding of EP risk can help prevent its occurrence. We carried out a multi-center, large-sample, case-control study to evaluate the risk factors for EP in Shanghai, China.

Methods: Women who were diagnosed with EP $(n=2411)$ and women with intrauterine pregnancies $(n=2416)$ were recruited from five hospitals in Shanghai, China. Information regarding the sociodemographic characteristics; reproductive, gynecological and surgical history; and previous and current use of contraceptives was collected from all participants. Odds ratios (ORs) and $95 \%$ confidence intervals (Cls) were calculated and adjusted for potential confounding factors via multivariate logistic regression analysis.
\end{abstract}

Results: The study revealed that the risk of EP was associated with the traditional risk factors including previous EP (Adjusted odds ratio $[\mathrm{AOR}]=2.72,95 \% \mathrm{Cl}: 1.83-4.05$ ), previous Chlamydia trachomatis infection (Adjusted $\mathrm{OR}=3.18$, $95 \% \mathrm{Cl}: 2.64,3.84)$, previous infertility $(\mathrm{AOR}=2.18,95 \% \mathrm{Cl}: 1.66-2.88)$, previous adnexal surgery $(\mathrm{AOR}=2.09,95 \%$ Cl: 1.49-2.93), previous appendectomy ( $\mathrm{AOR}=1.64,95 \% \mathrm{Cl}: 1.13-2.37)$, and previous use of intrauterine devices (IUDs) (AOR $=1.72,95 \% \mathrm{Cl}: 1.39-2.13)$. Additionally, EP risk was increased following the failure of most contraceptives used in the current cycle including IUDs (AOR $=16.43,95 \% \mathrm{Cl}: 10.42-25.89$ ), oral contraceptive pills $(\mathrm{AOR}=3.02,95 \% \mathrm{Cl}:$ 1.16-7.86), levonorgestrel emergency contraception ( $\mathrm{OOR}=4.75,95 \% \mathrm{Cl}: 3.79-5.96)$, and female sterilization ( $A O R=4.73,95 \% \mathrm{Cl}: 1.04-21.52$ ). Stratified analysis showed that in vitro fertilization and embryo transfer (IVF-ET) was the main risk factor for EP in women with tubal infertility (AOR $=8.99,95 \%$ Cl: 1.98-40.84), although IVF-ET showed no association with EP in women with non-tubal infertility ( $A O R=2.52,95 \%$ Cl: 0.14-44.67).

Conclusion: In addition to the traditional risk factors, IVF-ET and current IUD use play dominant roles in the occurrence of EP. Attention should be given to women with tubal infertility who have undergone IVE-ET treatment.

\section{Background}

Ectopic pregnancy (EP) is the leading cause of maternal death during the first trimester of pregnancy, accounting for approximately $10 \%$ of all pregnancy-related deaths [1]. It remains to be a condition presenting as a serious health problem for women of childbearing age [2]. It has been shown to reduce subsequent fertility and increase the chances of subsequent EP [3]. Over recent decades, there has been a rise in the incidence of EP [4].

\footnotetext{
*Correspondence: zhangjian_ipmch@sjtu.edu.cn; qjchildren736@qq.com ${ }^{\dagger}$ Equal contributors

'Department of Obstetrics and Gynecology, International Peace Maternity and Child Health Hospital, School of Medicine, Shanghai Jiao Tong University, Shanghai 200030, China

Full list of author information is available at the end of the article
}

There is extensive literature regarding the potential risk factors for EP. The identified risk factors for EP include age, previous EP, previous pelvic surgery, use of intrauterine devices (IUDs), female sterilization, history of pelvic inflammatory disease, history of infertility and smoking at the time of conception [5-12]. The increased awareness and knowledge on the risk factors for EP could enable an early and accurate diagnosis of the disease, resulting in a reduced need for surgery and fewer complications.

However, the study designs of previous researches focused on women not using contraception at the time of conception to explore the risk factors for EP comprehensively, which failed to evaluate the association 
between EP and contraceptive using in the current cycle of conception and might make the results in an overall ambiguity [7], because fertility intention might have a great impact on pregnancy outcome when study the risk factors for EP [13]. Furthermore, with the increased incidence of EP, and variance in population structure and regional differences, the risk factors of EP may have changed. Additionally, due to the increased incidence in infertility, currently approaches such as assisted reproduction technology (ART) are more widely used; therefore, their role and strength in the incidence of EP should be re-evaluated [14, 15]. Different from the previous study which investigated the risk factors for EP in women with planned pregnancy only in one hospital in Shanghai [16], the present was designed to conduct in five hospitals covering the population across urban and rural areas of Shanghai with a relatively good representation of the population, in order to comprehensively evaluate all the risk factors for EP among the general population, rather than those with planned pregnancy.

\section{Methods}

\section{Study design and participants}

This case-control study was conducted at five medical hospitals in Shanghai (two general hospitals and three maternity hospitals). The study protocol was approved by the Institutional Review Board of all the hospitals (International Peace Maternity and Child Health Hospital, Shanghai First People's Hospital, Songjiang Central Hospital, Songjiang Maternity and Child Health Hospital, and Minhang Central Hospital). Written informed consents were obtained from all the study participants before they were interviewed.

From March 2011 and April 2013, women who had been diagnosed with EPs in the inpatient gynecology department of each hospital were interviewed as potential candidates for the case group (EP group). Women with intrauterine pregnancy (IUP) at the prenatal and family planning clinics of the same hospitals matched for age ( \pm 5 years), marital status and gestational age ( \pm 7 days) at a ratio of 1:1 were included in the study as controls. The inclusion criteria of the study subjects were as described in our previous study [17].

\section{Data collection and variable specification}

The definitions of previous and current use of contraceptive methods have been described in our previous study [17].

All participants were interviewed via a questionnaire according to a standard protocol to obtain information on sociodemographic characteristics (age, marital status, education, birthplace, personal annual income, smoking and institutions); reproductive, gynecological and surgical history (including number of previous abortions, parity, history of previous EP, previous infertility, categories of infertility, ARTs applied in the current cycle of conception, previous Cesarean section, previous adnexal surgery, specific adnexal surgery, previous appendectomy); previous use of contraceptives (including levonorgestrel emergency contraception [LNG-EC]; IUDs; oral contraceptive pills [OCPs]; and other methods such as condoms, withdrawal method and calendar rhythm method), and current use of contraceptives (IUDs; OCPs; LNG-EC; female sterilization; and other methods such as condoms, withdrawal method and calendar rhythm method).

Serum Chlamydia trachomatis (CT) IgG antibodies were detected using enzyme-linked immunosorbent assay (ELISA; Beijing Biosynthesis Biotechnology, China) after collecting 5-mL blood samples from each participant.

\section{Statistical analysis}

We examined the frequency distribution of each variable according to the case and control groups. Univariate logistic regression analysis was used to estimate the crude odds ratios (ORs) of each variable and their $95 \%$ confidence intervals (CIs). The variables associated with EP by univariate analysis were included as candidates in the multivariable logistic regression analysis by stepwise selection with a SLENTRY level of 0.1 and SLSTAY level of 0.1 .

When we explored the association between the risk of EP and ART, history of infertility was a major confounding factor. Thus, the study participants with history of infertility were divided into two strata, women with tubal infertility and those with non-tubal infertility. The association between risk of EP and each ART were analyzed in both strata. ORs and their $95 \%$ CIs were calculated and adjusted for other potential confounding factors, including age (less than 20, 20-24, 25-30, 30-34, 35-39, or greater than 40 years of age), medical hospitals (1, 2, 3 , 4 or 5), educational level (primary school or less, middle school, high school or college or higher degree), occupation (employed, self-employed or unemployed), previous EP (no or yes), serum CT IgG test (negative or positive), previous adnexal surgery (no or yes), previous appendectomy (no or yes), previous use of IUDs (no or yes), previous use of other contraceptive methods (no or yes), and current use of contraceptive methods (not used, OCPs, LNG-EC, IUDs, female sterilization or other contraceptive methods).

All statistical analyses were performed using SAS software, version 8.2 (SAS Institute, Inc., Cary, NC). All $p$ values were calculated using two-sided tests. Differences between values were considered statistically significant at a $p$ value of less than 0.05 . 


\section{Results}

From the study subjects, 148 EP and 118 IUP patients either refused to participate in the interview or provided incomplete information in the questionnaire survey; these women were excluded from the study. Finally, 2411 EP patients and 2416 IUP controls were included in this study, and the response rate was $94.78 \%$ (Fig. 1).

\section{Univariate analysis}

Table 1 presents the distribution of sociodemographic characteristics between both the groups. EP patients were more likely to be born out of Shanghai $\left(p<10^{-3}\right)$, have lower education attainment $\left(p<10^{-3}\right)$, and be self-employed/unemployed $\left(p<10^{-3}\right)$. However, smoking showed no relevance with EP (occasional smoker: $\mathrm{OR}=1.10,95 \% \mathrm{CI}: 0.77-1.59$; regular smoker: $\mathrm{OR}=$ 1.47, 95 \% CI: $0.95-2.28 ; p=0.41$ ). Due to the matching criteria of cases and controls in each hospital, there showed no significant difference in age $(p=0.16)$, marital status $(p=0.56)$ and institutions $(p=1.00)$ between the two groups.

Table 2 revealed the results of the analyses of crude association between the risk of EP and history of reproduction, gynecology and surgery. The occurrence of EP were showed to have a crude association with some factors including parity (once: OR $=1.14,95 \%$ CI: 1.02-1.30; more than twice: $\mathrm{OR}=1.58,95 \% \mathrm{CI}$ : 1.27-1.96), previous EP (OR $=6.67,95 \%$ CI: 5.049.11), previous $\mathrm{CT}$ infection $(\mathrm{OR}=3.83,95 \% \mathrm{CI}$ : $3.27-4.48)$, history of infertility $(\mathrm{OR}=4.42,95 \% \mathrm{CI}$ : 3.53-5.53), in vitro fertilization and embryo transfer (IVF-ET; OR $=5.01,95 \%$ CI: $1.53-16.44$ ), previous adnexal surgeries $(\mathrm{OR}=5.42,95 \% \mathrm{CI}: 4.29-6.84)$, and previous appendectomy $(\mathrm{OR}=1.67,95 \% \mathrm{CI}: 1.21-2.31)$.

In terms of the contraceptive experience (Table 3), previous IUD use was also associated with a higher risk of EP

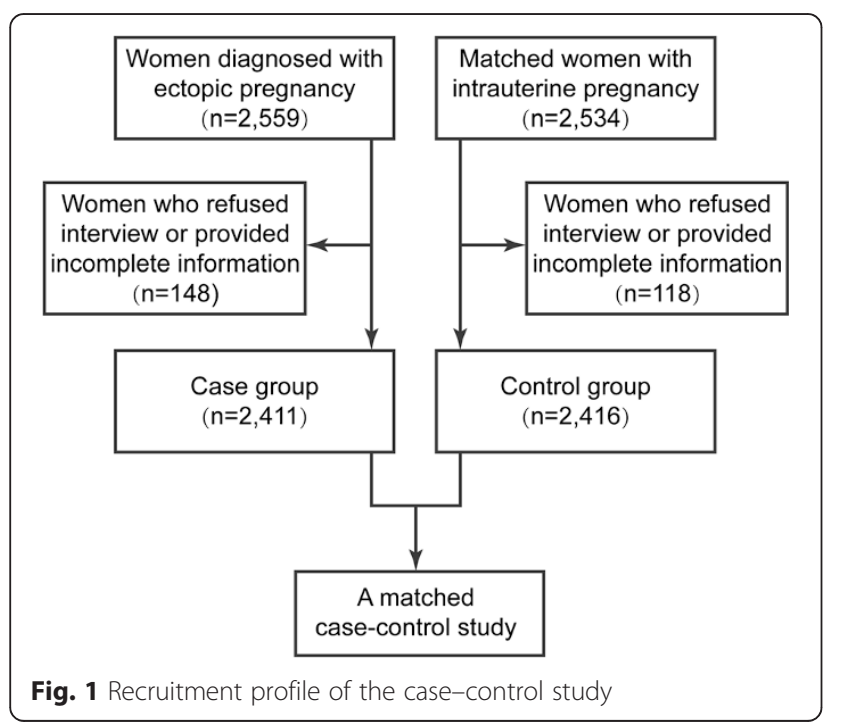

with an OR of 1.48 (95\% CI: 1.25-1.74), whereas previous use of other methods including condom, rhythm method and withdrawal method was associated with a lower risk $(\mathrm{OR}=0.39$, 95 \% CI: 0.34-0.45). Furthermore, a crude association was found between current use of most contraceptives and risk of EP (OCPs: $\mathrm{OR}=2.71,95 \% \mathrm{CI}$ : 1.11-6.61; LNG-EC: OR = 2.79, 95 \% CI: 2.27-3.43; IUDs: $\mathrm{OR}=11.41,95 \% \mathrm{CI}: 7.45-17.48$; female sterilization: $\mathrm{OR}=12.45,95$ \% CI: 2.91-53.18).

\section{Multivariate analysis}

Table 4 shows the results of the multivariate analysis between the risk of EP and candidate risk factors. Poor education and occupation were found to be independently associated with the risk of EP. The results revealed that women with previous EP (adjusted OR $[\mathrm{AOR}]=2.72$, $95 \%$ CI: $1.83-4.05)$, previous CT infection $(\mathrm{AOR}=3.18$, $95 \%$ CI: $2.64-3.84)$, a history of infertility $(\mathrm{AOR}=2.18$, 95 \% CI: 1.66-2.88), previous adnexal surgery (AOR = 2.09, $95 \%$ CI: 1.49-2.93), and previous appendectomy $(\mathrm{AOR}=1.64,95 \% \mathrm{CI}: 1.13-2.37)$ were at a greater risk of having an EP. With regards to contraception, previous IUD use was found to slightly increase the risk of EP $(\mathrm{AOR}=1.72,95 \% \mathrm{CI}: 1.39-2.13)$, while previous use of other contraceptive methods including condom, rhythm method and withdrawal method were shown to protect women from the incidence of $\mathrm{EP}(\mathrm{AOR}=0.56,95 \% \mathrm{CI}$ : $0.47-0.66$ ). In addition, current use of most contraceptives was significantly correlated with the incidence of EP following contraceptive failure, and the risk varied across the different contraceptive methods (OCPs: $\mathrm{AOR}=3.02$, 95 \% CI: 1.16-7.86; LNG-EC: AOR $=4.75,95$ \% CI: 3.795.96; IUDs: $A O R=16.43,95 \%$ CI: 10.42-25.89; female sterilization: $\mathrm{AOR}=4.73,95 \% \mathrm{CI}$ : 1.04-21.52). Notably, among women with a history of infertility, those who resorted to IVF-ET in the current cycle of conception showed a higher risk of EP (AOR = 9.28, 95 \% CI: 2.1440.38) than those who got spontaneously pregnant, while the use of Chinese herbal medicine and other ART approaches was not associated with the risk of EP (Chinese herbal medicine: $\mathrm{OR}=0.80,95 \%$ CI: 0.41-1.56; other ARTs: OR $=0.8,95 \%$ CI: 0.34-1.88).

Table 5 presents a stratified analysis of the association between EP risk and the ARTs applied in the current conception cycle, according to the different categories of infertility. In women with tubal infertility, IVF-ET was shown to significantly increase the risk of EP (AOR = 8.99, 95 \% CI: 1.98-40.84). However, there were no significant associations between the risk of EP and IVF-ET among women with non-tubal infertility $(\mathrm{AOR}=2.52$, 95 \% CI: 0.14-44.67). The risk of EP among women using Chinese herbs and other ARTs remained the same as that before stratification. 
Table 1 Socio-demographic characteristics of all enrolled participants

\begin{tabular}{|c|c|c|c|c|c|c|}
\hline & \multicolumn{2}{|l|}{ EP } & \multicolumn{2}{|l|}{ IUP } & \multirow[t]{2}{*}{ OR $[95 \% \mathrm{Cl}]$} & \multirow[t]{2}{*}{$p$ value } \\
\hline & $\overline{n^{a}}$ & $(\%)$ & $\overline{n^{a}}$ & (\%) & & \\
\hline \multicolumn{7}{|l|}{ Age (year) } \\
\hline$\leq 20$ & 24 & $(1.00)$ & 32 & $(1.33)$ & Reference & \multirow[t]{6}{*}{0.16} \\
\hline $20-24$ & 363 & $(15.06)$ & 398 & $(16.47)$ & $1.22[0.70,2.10]$ & \\
\hline $25-29$ & 753 & (31.23) & 772 & $(31.95)$ & $1.30[0.76,2.23]$ & \\
\hline $30-34$ & 793 & (32.89) & 755 & $(31.25)$ & $1.40[0.82,2.40]$ & \\
\hline $35-39$ & 332 & (13.77) & 322 & $(13.33)$ & $1.38[0.79,2.39]$ & \\
\hline$\geq 40$ & 146 & $(6.06)$ & 137 & $(5.67)$ & $1.42[0.80,2.53]$ & \\
\hline \multicolumn{7}{|l|}{ Marital status } \\
\hline Married & 2067 & $(85.80)$ & 2088 & $(86.42)$ & Reference & \multirow[t]{2}{*}{0.56} \\
\hline Unmarried & 342 & $(14.20)$ & 328 & (13.58) & $0.92[0.78,1.08]$ & \\
\hline \multicolumn{7}{|l|}{ Birth place } \\
\hline Shanghai & 698 & $(28.95)$ & 775 & (32.08) & Reference & \multirow[t]{2}{*}{$<10^{-3}$} \\
\hline Outside of Shanghai & 1713 & (71.05) & 1641 & $(67.92)$ & $1.16[1.03,1.31]$ & \\
\hline \multicolumn{7}{|l|}{ Education attainment } \\
\hline Collage or above & 1061 & $(44.01)$ & 1378 & $(57.04)$ & Reference & \multirow[t]{4}{*}{$<10^{-3}$} \\
\hline High school & 314 & (13.02) & 280 & (11.59) & $1.46[1.22,1.74]$ & \\
\hline Middle school & 178 & $(7.38)$ & 195 & $(8.07)$ & $1.19[0.95,1.48]$ & \\
\hline Primary school or lower & 858 & (35.59) & 563 & $(23.30)$ & $1.98[1.73,2.26]$ & \\
\hline \multicolumn{7}{|l|}{ Occupation } \\
\hline Employed & 1682 & $(69.88)$ & 1897 & (78.58) & Reference & \multirow[t]{3}{*}{$<10^{-3}$} \\
\hline Self-employed & 262 & $(10.89)$ & 184 & $(7.62)$ & $1.61[1.32,1.96]$ & \\
\hline Unemployed & 463 & $(19.24)$ & 333 & $(13.80)$ & $1.57[1.34,1.83]$ & \\
\hline \multicolumn{7}{|c|}{ Personal annual income (RMB) } \\
\hline$<50,000$ & 1165 & $(48.32)$ & 1093 & $(45.24)$ & Reference & \multirow[t]{3}{*}{0.13} \\
\hline $50,000-100,000$ & 777 & $(32.23)$ & 841 & $(34.81)$ & $0.87[0.76,0.99]$ & \\
\hline$>100,000$ & 469 & $(19.45)$ & 482 & $(19.95)$ & $0.91[0.79,1.06]$ & \\
\hline \multicolumn{7}{|l|}{ Smoking ${ }^{b}$} \\
\hline None smoker & 2298 & $(95.31)$ & 2294 & $(96.18)$ & Reference & \multirow[t]{3}{*}{0.41} \\
\hline Occasional smoker & 63 & $(2.61)$ & 57 & $(2.39)$ & $1.10[0.77,1.59]$ & \\
\hline Regular smoker & 50 & $(2.07)$ & 34 & $(1.43)$ & $1.47[0.95,2.28]$ & \\
\hline \multicolumn{7}{|l|}{ Institutions $^{c}$} \\
\hline 1 & 1404 & $(58.23)$ & 1409 & $(58.32)$ & Reference & \multirow[t]{5}{*}{1.00} \\
\hline 2 & 276 & $(11.45)$ & 274 & $(11.34)$ & $1.01[0.84,1.21]$ & \\
\hline 3 & 291 & $(12.07)$ & 293 & (12.13) & $1.00[0.83,1.19]$ & \\
\hline 4 & 272 & $(11.28)$ & 272 & $(11.26)$ & $1.00[0.84,1.21]$ & \\
\hline 5 & 168 & $(6.97)$ & 168 & $(6.95)$ & $1.00[0.80,1.26]$ & \\
\hline
\end{tabular}

$E P$ ectopic pregnancy, IUP intrauterine pregnancy, $O R$ odds ratio, $\mathrm{Cl}$ confidence interval

${ }^{a}$ The sum does not necessarily equal the sample size for all variables because of missing data

boccasional smoker: cigarette smoking more than 4 times a week, but a day on average less than 1 cigarette. Regular smoker: cigarette smoking more than 1 cigarettes per day, continuous or accumulated 6 months

'Center $1=$ International Peace Maternity and Child Health Hospital; Center $2=$ Shanghai First People's Hospital; Center $3=$ Songjiang Central Hospital;

Center $4=$ Songjiang Maternity and Child Health Hospital; Center 5 = Minhang Central Hospital

\section{Discussion}

Here, IVF-ET and current IUD use were found to be high-risk factors associated with the incidence of EP in
China. It has been suggested that tubal factor infertility rather than IVF-ET contributes to EP risk in women who undergo IVE-ET. Furthermore, other traditional 
Table 2 History of reproduction, gynecology, and surgery of all enrolled participates

\begin{tabular}{|c|c|c|c|c|c|c|}
\hline & \multicolumn{2}{|l|}{ EP } & \multicolumn{2}{|l|}{ IUP } & \multirow[t]{2}{*}{ OR $[95 \% \mathrm{Cl}]$} & \multirow[t]{2}{*}{$p$ value } \\
\hline & $n^{a}$ & (\%) & $n^{a}$ & (\%) & & \\
\hline \multicolumn{7}{|l|}{ Reproductive history } \\
\hline \multicolumn{7}{|l|}{ Number of previous abortions } \\
\hline 0 & 873 & $(36.88)$ & 930 & (38.49) & Reference & \multirow[t]{4}{*}{0.71} \\
\hline 1 & 763 & $(32.23)$ & 756 & $(31.29)$ & $1.08[0.94,1.23]$ & \\
\hline 2 & 485 & $(20.49)$ & 497 & $(20.57)$ & $1.04[0.89,1.21]$ & \\
\hline$\geq 3$ & 246 & (10.39) & 233 & $(9.64)$ & $1.13[0.92,1.38]$ & \\
\hline \multicolumn{7}{|l|}{ Parity } \\
\hline 0 & 1143 & $(48.29)$ & 1280 & $(52.98)$ & Reference & \multirow[t]{3}{*}{$<10^{-3}$} \\
\hline 1 & 994 & $(41.99)$ & 973 & $(40.27)$ & $1.14[1.02,1.30]$ & \\
\hline$\geq 2$ & 230 & $(9.72)$ & 163 & $(6.75)$ & $1.58[1.27,1.96]$ & \\
\hline \multicolumn{7}{|l|}{ Gynecologic history } \\
\hline \multicolumn{7}{|l|}{ Previous EP } \\
\hline No & 2093 & $(86.81)$ & 2363 & $(97.81)$ & Reference & \multirow[t]{2}{*}{$<10^{-3}$} \\
\hline Yes & 318 & (13.19) & 53 & $(2.19)$ & $6.77[5.04,9.11]$ & \\
\hline \multicolumn{7}{|l|}{ Serum Chlamydia trachomatis IgG test } \\
\hline Negative & 1648 & (69.13) & 2099 & $(89.55)$ & Reference & \multirow[t]{2}{*}{$<10^{-3}$} \\
\hline Positive & 736 & $(30.87)$ & 245 & $(10.45)$ & $3.83[3.27,4.48]$ & \\
\hline \multicolumn{7}{|l|}{ Previous infertility } \\
\hline No & 2005 & $(83.26)$ & 2286 & $(95.65)$ & Reference & \multirow[t]{2}{*}{$<10^{-3}$} \\
\hline Yes & 403 & $(16.74)$ & 104 & $(4.35)$ & $4.42[3.53,5.53]$ & \\
\hline \multicolumn{7}{|l|}{ Categories of infertility } \\
\hline No & 2005 & $(83.26)$ & 2286 & $(95.65)$ & Reference & \multirow[t]{3}{*}{$<10^{-3}$} \\
\hline Tubal infertility & 326 & $(13.54)$ & 73 & $(3.05)$ & $5.09[3.92,6.61]$ & \\
\hline Non-tubal infertility & 77 & $(3.20)$ & 31 & $(1.30)$ & $2.83[1.86,4.32]$ & \\
\hline \multicolumn{7}{|c|}{ ARTs applied in the current cycle of conception ${ }^{\mathrm{b}}$} \\
\hline Spontaneous pregnancy & 278 & (68.98) & 72 & $(69.23)$ & Reference & \multirow[t]{4}{*}{$<10^{-3}$} \\
\hline IVF-ET & 58 & $(14.39)$ & 3 & $(2.88)$ & $5.01[1.53,16.44]$ & \\
\hline Other $\mathrm{ARTS}^{\mathrm{c}}$ & 46 & $(11.41)$ & 18 & $(17.31)$ & $0.66[0.36,1.21]$ & \\
\hline Chinese herb & 21 & $(5.21)$ & 11 & $(10.58)$ & $0.49[0.23,1.07]$ & \\
\hline \multicolumn{7}{|l|}{ Surgical history } \\
\hline \multicolumn{7}{|l|}{ Previous cesarean section $^{d}$} \\
\hline No & 691 & $(56.09)$ & 624 & $(54.74)$ & Reference & \multirow[t]{2}{*}{0.79} \\
\hline Yes & 541 & $(43.91)$ & 516 & $(45.26)$ & $0.95[0.81,1.11]$ & \\
\hline \multicolumn{7}{|l|}{ Previous adnexal surgery } \\
\hline No & 1985 & $(82.33)$ & 2322 & $(96.19)$ & Reference & $<10^{-3}$ \\
\hline Yes & 426 & $(17.67)$ & 92 & $(3.81)$ & $5.42[4.29,6.84]$ & \\
\hline Specific adnexal surgery ${ }^{e}$ & & & & & & \\
\hline Ovarian surgery & 64 & $(15.02)$ & 36 & (39.13) & $2.08[1.38,3.14]$ & $<10^{-3}$ \\
\hline Surgery for EP & 237 & $(55.62)$ & 38 & $(41.30)$ & $7.30[5.15,10.33]$ & \\
\hline Tubal reconstructive surgery & 90 & $(21.13)$ & 14 & $(15.22)$ & $7.52[4.27,13.25]$ & \\
\hline Female sterilization & 21 & $(4.93)$ & 2 & $(2.17)$ & $12.28[2.88,52.45]$ & \\
\hline Reversal of tubal sterilization & 14 & $(3.29)$ & 2 & $(2.17)$ & $8.19[1.86,36.07]$ & \\
\hline
\end{tabular}


Table 2 History of reproduction, gynecology, and surgery of all enrolled participates (Continued)

\begin{tabular}{llllll}
\hline Previous appendectomy & & & & \\
No & 2303 & $(95.84)$ & 2346 & $(97.47)$ & Reference \\
Yes & 100 & $(4.16)$ & 61 & $(2.53)$ & $1.67[1.21,2.31]$ \\
\hline
\end{tabular}

EP ectopic pregnancy, IUP intrauterine pregnancy, OR odds ratio, $C I$ confidence interval, $A R T$ assisted reproduction technology, IVF-ET in vitro fertilization and embryo transfer

${ }^{a}$ The sum does not necessarily equal the sample size for all variables because of missing data

${ }^{b}$ The number of women with history of infertile was used as the denomintor to calculate the percentage

'Other ARTs includes ovarian stimulation, intrauterine insemination, luteal phase support and combination of ovarian stimulation and luteal phase support

${ }^{d}$ The number of women having delivered a child was used as the denomintor to calculate the percentage

${ }^{\mathrm{e}}$ The number of women experienced adnexal surgeries was used as the denomintor to calculate the percentage

risk factors were still found to be associated with the incidence of EP.

The traditional risk factors for EP such as history of previous EP, previous adnexal surgery, previous appendectomy and CT infection have been well described elsewhere $[1,3,6,10]$. It is not surprising that the results of the current study are identical to previous reports of increased subsequent risk of EP by these traditional risk factors. This finding indicated that traditional risk factors still played a major role in the occurrence of EP.

Contraceptive failure is considered to be an important factor associated with the increased incidence of EP [18].
Due to the national family planning policy, there may be a difference in the contraception preferences in China [19]. All methods of contraception can effectively reduce the number of intrauterine and ectopic pregnancies. However, from our findings, when pregnancies occur as a result of contraceptive failure, the risk of ectopic pregnancy is significantly increased in women who become pregnant after tubal sterilization or after using IUDs, OCPs and LNG-EC. This finding from the present study was identical to the results from a meta-analysis [20]. Previous studies indicated that progesterone and its analogue, LNG, could effectively inhibit human tubal

Table 3 Previous and current use of contraception

\begin{tabular}{|c|c|c|c|c|c|c|}
\hline & \multicolumn{2}{|l|}{ EP } & \multicolumn{2}{|l|}{ IUP } & \multirow[t]{2}{*}{ OR $[95 \% \mathrm{Cl}]$} & \multirow[t]{2}{*}{$p$ value } \\
\hline & $\overline{n^{a}}$ & (\%) & $\overline{n^{a}}$ & (\%) & & \\
\hline \multicolumn{7}{|l|}{ Previous use of contraceptives } \\
\hline \multicolumn{7}{|l|}{ LNG-EC } \\
\hline No & 1283 & $(53.57)$ & 1311 & $(54.74)$ & Reference & \multirow[t]{2}{*}{0.53} \\
\hline Yes & 1112 & $(46.43)$ & 1084 & $(45.26)$ & $1.05[0.94,1.17]$ & \\
\hline \multicolumn{7}{|l|}{ IUDs } \\
\hline No & 1993 & $(83.74)$ & 2106 & $(88.27)$ & Reference & \multirow[t]{2}{*}{$<10^{-3}$} \\
\hline Yes & 387 & $(16.26)$ & 280 & $(11.74)$ & $1.48[1.25,1.74]$ & \\
\hline \multicolumn{7}{|l|}{ OCPs } \\
\hline No & 2254 & $(94.47)$ & 2295 & $(95.55)$ & Reference & \multirow[t]{2}{*}{0.06} \\
\hline Yes & 132 & $(5.53)$ & 107 & $(4.45)$ & $1.25[0.97,1.63]$ & \\
\hline \multicolumn{7}{|l|}{ Other contraceptive methods ${ }^{b}$} \\
\hline No & 829 & $(35.32)$ & 421 & $(17.50)$ & Reference & \multirow[t]{2}{*}{$<10^{-3}$} \\
\hline Yes & 1518 & $(64.68)$ & 1985 & $(82.50)$ & $0.39[0.34,0.45]$ & \\
\hline \multicolumn{7}{|l|}{ Current contraceptive methods } \\
\hline No & 1337 & $(55.87)$ & 1585 & $(65.60)$ & Reference & \multirow[t]{6}{*}{$<10^{-3}$} \\
\hline Other contraceptive methods ${ }^{b}$ & 447 & $(18.68)$ & 653 & $(27.03)$ & $0.81[0.71,0.93]$ & \\
\hline OCPs & 16 & $(0.67)$ & 7 & $(0.29)$ & $2.71[1.11,6.61]$ & \\
\hline LNG-EC & 341 & $(14.25)$ & 145 & $(6.00)$ & $2.79[2.27,3.43]$ & \\
\hline IUDs & 231 & $(9.65)$ & 24 & $(0.99)$ & $11.41[7.45,17.48]$ & \\
\hline Female sterilization ${ }^{c}$ & 21 & $(0.88)$ & 2 & $(0.08)$ & $12.45[2.91,53.18]$ & \\
\hline
\end{tabular}

EP ectopic pregnancy, IUP intrauterine pregnancy, OR odds ratio, $C$ confidence interval, IUDs intrauterine devices, LNG-EC levonorgestrel emergency contraception, OCPs oral contraceptive pills

${ }^{a}$ The sum does not necessarily equal the sample size for all variables because of missing data

${ }^{b}$ Other contraceptive methods includes condom, rhythm method, withdrawal

${ }^{c}$ Women who received reversal of tubal sterilization $(n=16)$ are not included here 
Table 4 Multivariable logistic regression analysis predicting risk factors for ectopic pregnancy

\begin{tabular}{lll}
\hline & AOR $[95 \% \mathrm{Cl}]$ & $p$ value \\
\hline Education attainment & & $<10^{-3}$ \\
Collage or above & Reference & \\
High school & $1.21[0.97,1.50]$ & \\
Middle school & $0.96[0.74,1.24]$ & \\
Primary school or lower & $1.47[1.23,1.75]$ & \\
Occupation & & $<10^{-2}$ \\
Employed & Reference & \\
Self-employed & $1.30[1.02,1.66]$ & \\
Unemployed & $1.33[1.10,1.62]$ & \\
Previous ectopic pregnancy & & $<10^{-3}$ \\
No & Reference & \\
Yes & $2.72[1.83,4.05]$ & \\
Serum Chlamydia trachomatis lgG test & & $<10^{-3}$ \\
Negative & Reference \\
Positive & $3.18[2.64,3.84]$ & \\
Previous infertility & & \\
No & Reference & \\
Yes & $2.18[1.66,2.88]$ &
\end{tabular}

ART applied in the current cycle of conception

$\begin{array}{ll}\text { Spontaneous pregnancy } & \text { Reference } \\ \text { IVF-ET } & 9.28[2.14,40.38] \\ \text { Other ARTs } & \\ \text { Chinese herb } & 0.80[0.34,1.88] \\ & 0.80[0.41,1.56]\end{array}$

Previous adnexal surgery

No

Yes

Reference

$2.09[1.49,2.93]$

Previous appendectomy

$\begin{array}{ll}\text { No } & \text { Reference } \\ \text { Yes } & 1.64[1.13,2.37]\end{array}$

Previous use of IUDs

No

Yes

Reference

$1.72[1.39,2.13]$

Previous use of other contraceptive methods ${ }^{b}$

$\begin{array}{ll}\text { No } & \text { Reference } \\ \text { Yes } & 0.56[0.47,0.66]\end{array}$

Current contraceptive methods

$\begin{array}{ll}\text { No } & \text { Reference } \\ \text { Other contraceptive methods }^{\mathrm{b}} & 1.22[0.99,1.44] \\ \text { OCPs } & 3.02[1.16,7.86]\end{array}$

$<10^{-3}$

fable 4 Multivariable logistic regression
factors for ectopic pregnancy (Continued)

\begin{tabular}{ll}
\hline LNG-EC & $4.75[3.79,5.96]$ \\
IUDs & $16.43[10.42,25.89]$ \\
Female sterilization & $4.73[1.04,21.52]$ \\
\hline
\end{tabular}

$A O R$ adjusted odds ratio, $\mathrm{Cl}$ confident interval, $A R T$ assisted reproduction technology, IVF-ET in vitro fertilization and embryo transfer, IUDs intrauterine devices, LNG-EC levonorgestrel emergency contraception, OCPs oral contraceptive pills

${ }^{a}$ Other ARTs includes ovarian stimulation, intrauterine insemination, luteal phase support and combination of ovarian stimulation and luteal phase support

bother contraceptive methods includes condom, rhythm method, withdrawal

activities [21, 22], which has been considered as the main cause of impaired embryo-tubal retention and implantation [23]. Although we failed to obtain the information on the type of OCPs, women following OCPs failure still should be aware of the possibilities of EP.

The results of this study were consistent with those of previous studies, which showed that a history of infertility was a risk factor for EP [7, 9, 24]. It has been acknowledged that IVF-ET is a valuable treatment for infertility, especially for the treatment of tubal infertility. Ever since the first pregnancy conceived following IVFET treatment in 1976 was an ectopic one [25], the association between EP risk and ARTs has been debated [26]. The incidence of EP following ARTs, especially IVF-ET, has been reported to reduce since 2001 [27]. However, another study investigated the incidence of EP in 128,314 pregnancies following ARTs according to the presence or absence of tubal infertility, and concluded that the incidence of EP following ARTs was higher in women with tubal infertility than in women without tubal infertility [28]. In addition, Strandell et al. suggested that tubal infertility was the most prominent risk factor for EP following IVF-ET [29]. However, our previous study failed to explore whether ART have an influence on the risk of EP, due to the small number of women with non-tubal infertility becoming pregnant with ARTs [16]. Based on this large multi-center study, the stratification analysis indicated that women with tubal infertility and not those with non-tubal infertility were at a greater risk of EP when they underwent IVF-ET in their current conception cycle. Therefore, the present study indicated that it was IVF-ET that contributed to the risk of EP for women who underwent IVF-ET.

The present study has some limitations. Data collection in this study was based on patients' self-evaluation; hence, we were unable to obtain additional information on the types of OCPs, sterilization methods and IUDs for further study. In addition, a limited number of women with nontubal infertility underwent IVF-ET to become pregnant; thus, further prospective cohort studies are needed to verify our findings on the association between EP and 
Table 5 The association between EP and the mode of current pregnancy among women with tubal infertility or non-tubal infertility

\begin{tabular}{|c|c|c|c|c|c|c|c|c|c|c|}
\hline & \multicolumn{5}{|c|}{ Tubal infertility $^{a}$} & \multicolumn{5}{|c|}{ Non-tubal infertility ${ }^{b}$} \\
\hline & \multicolumn{2}{|l|}{ EP } & \multicolumn{2}{|l|}{ IUP } & \multirow[t]{2}{*}{$\mathrm{AOR}[95 \% \mathrm{Cl}]^{d}$} & \multicolumn{2}{|l|}{ EP } & \multicolumn{2}{|l|}{ IUP } & \multirow[t]{2}{*}{ AOR $[95 \% \mathrm{Cl}]^{\mathrm{d}}$} \\
\hline & $n^{c}$ & (\%) & $n^{c}$ & (\%) & & $n^{c}$ & $(\%)$ & $n^{c}$ & (\%) & \\
\hline \multicolumn{11}{|c|}{ ARTs applied in the current cycle of conception } \\
\hline Spontaneous pregnancy & 223 & $(68.41)$ & 50 & $(68.49)$ & Reference & 55 & $(71.43)$ & 22 & $(70.97)$ & Reference \\
\hline IVF-ET & 55 & $(16.87)$ & 2 & $(2.74)$ & $8.99[1.98,40.84]$ & 3 & $(3.90)$ & 1 & $(3.23)$ & $2.52[0.14,44.67]$ \\
\hline Other ARTs ${ }^{\mathrm{e}}$ & 29 & $(8.9)$ & 12 & $(16.44)$ & $0.59[0.25,1.39]$ & 17 & $(22.08)$ & 6 & (19.36) & $0.94[0.22,3.98]$ \\
\hline Chinese herb & 19 & $(5.83)$ & 9 & (12.33) & $0.78[0.31,2.01]$ & 2 & $(2.60)$ & 2 & $(6.45)$ & $0.35[0.03,4.50]$ \\
\hline
\end{tabular}

EP ectopic pregnancy, IUP intrauterine pregnancy, AOR adjusted odds ratio, $C l$ confident interval, IVF-ET in vitro fertilization and embryo transfer, ART assisted reproduction technology

${ }^{\text {aT }}$ This analysis was restricted to 399 people with tubal infertility

${ }^{\mathrm{b}}$ This analysis was restricted to 108 people with non-tubal infertility

${ }^{\mathrm{C}}$ The sum does not necessarily equal the sample size for all variables because of missing data

${ }^{\mathrm{d} O d d s}$ ratios were adjusted for age, institutions, education attainment, occupation, previous EP, serum CT IgG test, previous adnexal surgery, previous appendectomy, previous use of IUDs, previous use of other contraceptive methods and current contraceptive methods

e Other ARTs includes Ovarian stimulation, intrauterine insemination, luteal phase support and combination of ovarian stimulation and luteal phase support

IVF-ET among women with non-tubal infertility. Moreover, our data did not cover the technical and qualitative aspects of the IVF procedure (e.g., the stimulation protocols, endometrial and ovarian responses, embryo quality, transfer technique, number of embryos transferred and use of luteal support). Together, all these aspects may contribute to the risk of EP in women with IVF-ET [29]. As a hospital-based case-control study, recall and selection bias must be acknowledged. The multi-centered design carried out across five hospitals covering the urban and rural areas of Shanghai and the large sample size are the strengths of this study, and helped recruit a relatively good representation of the population thereby minimizing selection bias.

\section{Conclusion}

In this large multi-center case-control study, the risk of EP still show correlation with some traditional risk factors including previous EP, previous CT infection, previous infertility, previous adnexal surgery, previous use of IUDs and current use of IUDs, OCPs, LNG-EC and female sterilization. Although IVF-ET has been associated with an increased risk of EP incidence among women with a history of infertility, this was only observed among women with tubal infertility and not in women with non-tubal infertility. In general, physicians should pay attention to suspected EP cases with exposure to some traditional risk factors. Furthermore, attention of EP should also be paid to pregnant women following IVF-ET, particularly among tubal infertility cases.

\section{Abbreviations}

EP: Ectopic pregnancy; IUDs: Intrauterine devices; ART: Assisted reproduction technology; IUP: Intrauterine pregnancy; LNG-EC: Levonorgestrel emergency contraception; OCPs: Oral contraceptive pills; CT: Chlamydia trachomatis; ELISA: Enzyme-linked immunosorbent assay; OR: Odds ratio; Cl: Confidence interval; IVF-ET: In vitro fertilization and embryo transfer.

\section{Competing interests}

The authors declare that they have no competing interests.

\section{Authors' contributions}

$\mathrm{CL}$ conducted to the study design and drafted the manuscript. WHZ contributed to the interpretation of data and critical revision of the manuscript. QZ contributed to interpretation of data and critical revision of the manuscript. SJC, HP, XX, GJQ, MXY contributed to the data collection and carried out carried out the ELISA. DZ participated in the study design and performed the statistical analysis. JQ, JZ contributed to conceive of the study, and participated in its design and coordination and helped to draft the manuscript. All authors approved the final version of the manuscript.

\section{Acknowledgements}

This work was supported by a grant from the Shanghai Scientific and Technical Committee Foundation (124119a4802).

\section{Author details}

'Department of Obstetrics and Gynecology, International Peace Maternity and Child Health Hospital, School of Medicine, Shanghai Jiao Tong University, Shanghai 200030, China. ${ }^{2}$ Institute of Embryo-Fetal Original Adult Disease Affiliated to Shanghai Jiao Tong University School of Medicine, Shanghai 200030, China. ${ }^{3}$ Department of Obstetrics and Gynecology, Songjiang Central Hospital, Shanghai 201600, China. ${ }^{4}$ Department of Obstetrics and Gynecology, Songjiang Maternity and Child Health Hospital, Shanghai 201620, China. ${ }^{5}$ Department of Obstetrics and Gynecology, Shanghai First People's Hospital, Shanghai Jiao Tong University, Shanghai 200080, China. ${ }^{6}$ Department of Obstetrics and Gynecology, Minhang Central Hospital, Shanghai 201100, China.

Received: 25 January 2015 Accepted: 10 August 2015

Published online: 22 August 2015

\section{References}

1. Farquhar CM. Ectopic pregnancy. Lancet. 2005;366(9485):583-91.

2. Zane SB, Kieke BJ, Kendrick JS, Bruce C. Surveillance in a time of changing health care practices: estimating ectopic pregnancy incidence in the United States. Matern Child Health J. 2002;6(4):227-36.

3. Chow WH, Daling JR, Cates WJ, Greenberg RS. Epidemiology of ectopic pregnancy. Epidemiol Rev. 1987;9:70-94.

4. Walker JJ. Ectopic pregnancy. Clin Obstet Gynecol. 2007;50(1):89-99.

5. Yuk JS, Kim YJ, Hur JY, Shin JH. Association between socioeconomic status and ectopic pregnancy rate in the Republic of Korea. Int J Gynaecol Obstet. 2013;122(2):104-7.

6. Job-Spira N, Collet P, Coste J, Bremond A, Laumon B. Risk factors for ectopic pregnancy. Results of a case control study in the Rhone-Alpes region. Contracept Fertil Sex. 1993;21(4):307-12.

7. Bouyer J, Coste J, Shojaei T, Pouly JL, Fernandez H, Gerbaud L, et al. Risk factors for ectopic pregnancy: a comprehensive analysis based on a large 
case-control, population-based study in France. Am J Epidemiol. 2003;157(3):185-94.

8. Parashi S, Moukhah S, Ashrafi M. Main risk factors for ectopic pregnancy: a case-control study in a sample of Iranian women. Int J Fertil Steril. 2014;8(2):147-54

9. Moini A, Hosseini R, Jahangiri N, Shiva M, Akhoond MR. Risk factors for ectopic pregnancy: a case-control study. J Res Med Sci. 2014;19(9):844-9.

10. Ankum WM, Mol BW, Van der Veen F, Bossuyt PM. Risk factors for ectopic pregnancy: a meta-analysis. Fertil Steril. 1996;65(6):1093-9.

11. Kamwendo F, Forslin L, Bodin L, Danielsson D. Epidemiology of ectopic pregnancy during a 28 year period and the role of pelvic inflammatory disease. Sex Transm Infect. 2000;76(1):28-32.

12. Barnhart KT, Sammel MD, Gracia CR, Chittams J, Hummel AC, Shaunik A. Risk factors for ectopic pregnancy in women with symptomatic first-trimester pregnancies. Fertil Steril. 2006;86(1):36-43.

13. Weiss NS, Daling JR, Chow WH. Control definition in case-control studies of ectopic pregnancy. Am J Public Health. 1985;75(1):67-8.

14. Esmaeilzadeh S, Delavar MA, Zeinalzadeh M, Mir MR. Epidemiology of infertility: a population-based study in Babol, Iran. Women Health. 2012;52(8):744-54

15. Malak M, Tawfeeq T, Holzer H, Tulandi T. Risk factors for ectopic pregnancy after in vitro fertilization treatment. J Obstet Gynaecol Can. 2011;33(6):617-9.

16. Li C, Meng C, Zhao W, Lu H, Shi W, Zhang J. Risk factors for ectopic pregnancy in women with planned pregnancy: a case-control study. Eur J Obstet Gynecol Reprod Biol. 2014;181:176-82.

17. Li C, Zhao W, Meng C, Ping H, Qin G, Cao S, et al. Contraceptive Use and the Risk of Ectopic Pregnancy: A Multi-Center Case-Control Study. PLoS One. 2014;9(12):e115031.

18. Rana P, Kazmi I, Singh R, Afzal M, Al-Abbasi FA, Aseeri A, et al. Ectopic pregnancy: a review. Arch Gynecol Obstet. 2013;288(4):747-57.

19. Zheng X, Tan L, Ren Q, Cui Z, Wu J, Lin T, et al. Trends in contraceptive patterns and behaviors during a period of fertility transition in China: 1988-2006. Contraception. 2012;86(3):204-13.

20. Mol BW, Ankum WM, Bossuyt PM, Van der Veen F. Contraception and the risk of ectopic pregnancy: a meta-analysis. Contraception. 1995;52(6):337-41.

21. Wanggren K, Stavreus-Evers A, Olsson C, Andersson E, Gemzell-Danielsson K. Regulation of muscular contractions in the human Fallopian tube through prostaglandins and progestagens. Hum Reprod. 2008;23(10):2359-68.

22. Zhao W, Zhu Q, Yan M, Li C, Yuan J, Qin G, et al. Levonorgestrel decreased cilia beat frequency of human fallopian tubes and rat oviducts without changing the morphological structure. Clin Exp Pharmacol Physiol. 2014:42(2):171-8

23. Shaw JL, Dey SK, Critchley HO, Horne AW. Current knowledge of the aetiology of human tubal ectopic pregnancy. Hum Reprod Update. 2010;16(4):432-44

24. Karaer A, Avsar FA, Batioglu S. Risk factors for ectopic pregnancy: a case-control study. Aust N Z J Obstet Gynaecol. 2006;46(6):521-7.

25. Steptoe PC, Edwards RG. Reimplantation of a human embryo with subsequent tubal pregnancy. Lancet. 1976;1 (7965):880-2.

26. Chang HJ, Suh CS. Ectopic pregnancy after assisted reproductive technology: what are the risk factors? Curr Opin Obstet Gynecol. 2010;22(3):202-7.

27. Perkins KM, Boulet SL, Kissin DM, Jamieson DJ. Risk of ectopic pregnancy associated with assisted reproductive technology in the United States, 2001-2011. Obstet Gynecol. 2015;125(1):70-8.

28. Schippert C, Soergel P, Staboulidou I, Bassler C, Gagalick S, Hillemanns P, et al. The risk of ectopic pregnancy following tubal reconstructive microsurgery and assisted reproductive technology procedures. Arch Gynecol Obstet. 2012;285(3):863-71.

29. Strandell A, Thorburn J, Hamberger L. Risk factors for ectopic pregnancy in assisted reproduction. Fertil Steril. 1999;71(2):282-6.

\section{Submit your next manuscript to BioMed Central and take full advantage of:}

- Convenient online submission

- Thorough peer review

- No space constraints or color figure charges

- Immediate publication on acceptance

- Inclusion in PubMed, CAS, Scopus and Google Scholar

- Research which is freely available for redistribution 